\title{
Impulse lethal control and contraception control of seasonal breeding rodent
}

\author{
Hanwu Liu*, Fengqin Zhang and Qiuying Li
}

\author{
"Correspondence: \\ liuhanwu-china@163.com \\ Department of Applied \\ Mathematics, Yuncheng University, \\ Yuncheng, 044000, P.R. China
}

\begin{abstract}
The rodent is one important component of an ecosystem; however, abundance of rodents may have negative effects to humans. Based on a seasonally breeding model and the logistic model, the effect of impulse lethal control and impulse contraception control on rodent population dynamics is investigated. The existence and stability of the periodic solution were analyzed. The condition of rodent population dying out is the same for lethal and contraception control. However, the process of rodent population tending to a stable periodic solution is different obviously. Under lethal control, the rodent population tends to a stable periodic solution more quickly, whereas under contraception control, the rodent population develops slowly.
\end{abstract}

Keywords: seasonally breeding; impulse equation; periodic solution; contraception control; lethal control

\section{Introduction}

The rodent is one important component of an ecosystem, it is indispensable and irreplaceable. Due to the seasonal variation of food availability and physical environment, some species of rodents are seasonally breeding and their population sizes vary in a wavelike way. Examples of seasonally breeding rodents are listed in Table 1.

The amount of rodents may increase dramatically due to some reason and bring negative effects to humans. In practice, lethal control and contraception control are usually used to control overabundance of rodents. Lethal control is killing rodents by means of rodenticide or mousetrap and its aim is increasing the death rate of rodent population. Contraception control is preventing all or part of the rodent to produce normally by means of sterilant or special virus so as to reduce the birth rate of rodent population.

How the controlled rodent population develops is one important issue. Despite the fact that it is difficult to engage in this issue through experiments, mathematical modeling may be helpful. With the prerequisite of seasonally breeding, some discrete models have been formulated and analyzed to investigate the effect of lethal control or contraception control on population dynamics $[1,2]$.

In the present paper, based on seasonally breeding model and the logistic model, the effect of lethal control and contraception control on rodent population dynamics is investigated. In the seasonally breeding model, one year is divided into breeding season and non-breeding season and we describe the rodent population dynamics in these two time periods with different continuous mathematical models. When the long-term dynamics

(c) The Author(s) 2017. This article is distributed under the terms of the Creative Commons Attribution 4.0 International License (http://creativecommons.org/licenses/by/4.0/), which permits unrestricted use, distribution, and reproduction in any medium, provided you give appropriate credit to the original author(s) and the source, provide a link to the Creative Commons license, and indicate if changes were made. 
Table 1 Seasonally breeding rodent

\begin{tabular}{|c|c|c|c|}
\hline Species of rodent & Region & Breeding season & Reference \\
\hline Peromyscus & North America & Seasonally breeding & {$[3,4]$} \\
\hline Ochotona curzoniae & Heimahe, China & Seasonally breeding & {$[5]$} \\
\hline Arvicanthis niloticus & Oursi, Burkina Faso & Seasonally breeding & {$[6]$} \\
\hline \multicolumn{4}{|l|}{ Mastomys erythroleucus } \\
\hline \multicolumn{4}{|l|}{ Taterillus gracilis } \\
\hline \multicolumn{4}{|l|}{ Gerbillus nigeriae } \\
\hline \multicolumn{4}{|l|}{ Taterillus petteri } \\
\hline \multicolumn{4}{|l|}{ Acomys } \\
\hline Cryptomys hottentotus hottentotus & Western Cape, South Africa & September to November & [7] \\
\hline Cryptomys hottentotus pretoriae & Gauteng, South Africa & July to November & {$[8]$} \\
\hline Bathyergus janetta & Kamieskroon, South Africa & July to September & [9] \\
\hline Lophuromys flavopuncatus & Magamba, Tanzania & February to May & {$[10]$} \\
\hline \multicolumn{4}{|l|}{ Grammomys dolichurus } \\
\hline \multicolumn{4}{|l|}{ Praomys delectorum } \\
\hline Lasiopodomys brandtii & & March to September & {$[11]$} \\
\hline Meriones unguiculatus & Taipusi, China & Spring and summer & {$[12]$} \\
\hline Acomys dimidiatus & Taif, Saudi Arabia & Spring to Autumn & [13] \\
\hline Calomystener & Federal District, Brazil & Rainy season & [14] \\
\hline
\end{tabular}

of the rodent population is concerned, one year is only a time point, the seasonality of breeding is erased, and the population dynamics is described with the logistic model. Generically, lethal control is actualized through rodenticide and contraception control is actualized through sterilant. After ingesting bait that includes rodenticide or sterilant, the rodent would die or be sterile during a period not longer than several days. This period is very short compared to the whole lifetime of rodent, so we suppose that the control is accomplished at a time point instantaneously. Usually, there is a long time interval between two control practices, so we assume that the controls are implemented at discrete times with equal intervals.

\section{Logistic model and seasonally breeding model}

\subsection{Logistic model}

The logistic model is

$$
x^{\prime}=r x\left(1-\frac{x}{K}\right)
$$

where $x(t)$ is the population size at time $t, r>0$ is the intrinsic increasing rate and $K$ is the carrying capacity. The solution of the logistic model with initial condition $x\left(t_{0}\right)=x_{0}$ is $x(t)=\frac{K x_{0}}{x_{0}+\left(K-x_{0}\right) \mathrm{e}^{-r\left(t-t_{0}\right)}}$. For arbitrary $x_{0} \neq 0, \lim _{t \rightarrow+\infty} x(t)=K$, that is, the positive equilibrium $x=K$ is globally asymptotically stable (eliminate the case $x_{0}=0$ ).

\subsection{Seasonally breeding model}

For seasonally breeding rodents, assume that the breeding season and the non-breeding season are present in turn, the length of the breeding season is $T_{1}$, the length of the nonbreeding season is $T_{2}$, and the sum of $T_{1}$ and $T_{2}$ is one year, denoted by $T$. Let $x(t)$ be the size of the rodent population at time $t$. For convenience, let $t=0$ be the beginning of a breeding season. During the breeding season, $x(t)$ increases gradually, and beyond the breeding season $x(t)$ decreases gradually due to death. Assume that $x(t)$ satisfies the logistic model during the breeding season and $x(t)$ satisfies the Malthusian model during 
non-breeding season. Then one has

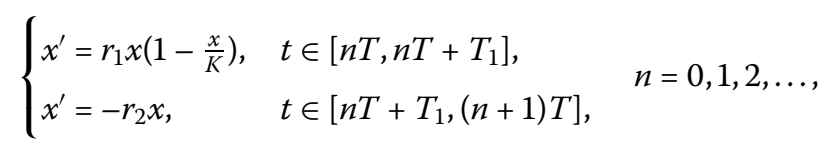

where $r_{1}>0$ is the intrinsic increasing rate during breeding season, $K$ is the carrying capacity, and $r_{2}>0$ is the death rate during non-breeding season. This model has been studied [15].

Lemma 1 is useful in studying the dynamical behavior of model (1).

Lemma 1 The sequence $\left\{y_{n}\right\}$ satisfies

$$
y_{n+1}=G\left(y_{n}\right)=\frac{a y_{n}}{b y_{n}+c}
$$

where $a, b, c$ are all positive constants. Then 0 is always an equilibrium of (2), when $a>$ $c$, (2) has another unique positive equilibrium $y^{*}=\frac{a-c}{b}$. There is no other $h(>1)$ periodic equilibrium at all. If $c \geq a$, then 0 is globally asymptotically stable and if $c<a$, then $y^{*}$ asymptotically attracts all positive solutions.

Proof Simple calculation gives the existence of equilibria. For positive integer $h>1, y_{n+h}=$ $G^{h}\left(y_{n}\right)=\frac{a^{h}(a-c) y_{n}}{b\left(a^{h}-c^{h}\right) y_{n}+c^{h}(a-c)}$ and it has no other equilibrium than 0 and $y^{*}$. That is, (2) has no other $h(>1)$ periodic equilibrium at all.

Obviously, if $y_{0}=0$, then $y_{n} \equiv 0$ and if $y_{0} \neq 0$, then, for any positive integer $n, y_{n} \neq 0$. When $y_{0} \neq 0$, rewrite equation (2) as

$$
\frac{1}{y_{n+1}}=\frac{b}{a}+\frac{c}{a} \cdot \frac{1}{y_{n}}
$$

When $a=c$, (3) is $\frac{1}{y_{n+1}}=\frac{b}{a}+\frac{1}{y_{n}}$, its solution is $y_{n}=\frac{1}{\frac{1}{y_{0}}+\frac{n b}{a}}$, so $\lim _{n \rightarrow+\infty} y_{n}=0$.

When $a \neq c$, (3) implies $\frac{1}{y_{n+1}}-\frac{b}{a-c}=\frac{c}{a}\left(\frac{1}{y_{n}}-\frac{b}{a-c}\right)$, its solution is $y_{n}=\frac{1}{\left(\frac{c}{a}\right)^{n}\left(\frac{1}{y_{0}}-\frac{b}{a-c}\right)+\frac{b}{a-c}}$. Thus, if $c>a$, then $\lim _{n \rightarrow+\infty} y_{n}=0$ and if $c<a$, then $\lim _{n \rightarrow+\infty} y_{n}=y^{*}$. The proof is complete.

Solving model (1) on time interval $[n T,(n+1) T]$, one has the mapping (4) from $x(n T)$ to $x((n+1) T)$,

$$
x((n+1) T)=G_{1}(x(n T))=\frac{K x(n T) \mathrm{e}^{-r_{2} T_{2}}}{\left(1-e^{-r_{1} T_{1}}\right) x(n T)+K \mathrm{e}^{-r_{1} T_{1}}} .
$$

Then Theorem 1 can be obtained from Lemma 1 immediately.

Theorem 10 is always an equilibrium of (4), if $r_{2} T_{2}<r_{1} T_{1}$, then (4) has another unique positive equilibrium $x_{1}^{*}=\frac{K\left(e^{-r_{2} T_{2}}-e^{-r_{1} T_{1}}\right)}{1-e^{-r_{1} T_{1}}}$. There is no other $h(>1)$ periodic equilibrium at all. If $r_{2} T_{2} \geq r_{1} T_{1}$, then 0 is globally asymptotically stable and model (1) has a globally asymptotically stable zero solution. If $r_{2} T_{2}<r_{1} T_{1}$, then $x_{1}^{*}$ asymptotically attracts all posi- 
tive solutions of (4) and model (1) has globally asymptotically stable periodic solution

$$
\left\{\begin{array}{ll}
x=\frac{K x_{1}^{*}}{x_{1}^{*}+\left(K-x_{1}^{*}\right) \mathrm{e}^{-r_{1}(t-n T)},} & t \in\left[n T, n T+T_{1}\right], \\
x=\frac{K x_{1}^{*} \mathrm{e}^{-r_{2}\left(t-n T-T_{1}\right)}}{x_{1}^{*}+\left(K-x_{1}^{*}\right) \mathrm{e}^{-r_{1} T_{1}}}, & t \in\left[n T+T_{1},(n+1) T\right],
\end{array} \quad n=0,1,2, \ldots\right.
$$

Obviously, when $r_{2} T_{2} \geq r_{1} T_{1}$, the rodent pest dies out, there is no damage. Thus rodent control is considered under the condition $r_{2} T_{2}<r_{1} T_{1}$ hereafter.

\section{Impulse lethal control}

\subsection{Logistic model with lethal control}

The logistic model with impulse lethal control is

$$
\begin{cases}x^{\prime}=r x\left(1-\frac{x}{K}\right), & t \neq n U \\ x\left(n U^{+}\right)=(1-p) x(n U), & t=n U\end{cases}
$$

where $p$ is the removal rate and lethal control is implemented once at regular time intervals $U$.

Solving model (5) on time interval $\left[n U^{+},(n+1) U^{+}\right]$, one has the mapping (6) from $x\left(n U^{+}\right)$ to $x\left((n+1) U^{+}\right)$,

$$
x\left((n+1) U^{+}\right)=G_{2}\left(x\left(n U^{+}\right)\right)=\frac{(1-p) K x\left(n U^{+}\right)}{\left(1-\mathrm{e}^{-r U}\right) x\left(n U^{+}\right)+K \mathrm{e}^{-r U}} .
$$

Then mapping (6) and Lemma 1 imply Theorem 2.

Theorem 20 is always an equilibrium of (6), when $p<1-\mathrm{e}^{-r U}$, then (6) has another unique positive equilibrium $x_{2}^{*}=\frac{\left(1-e^{-r U}-p\right) K}{1-e^{-r U}}$. There is no other $h(>1)$ periodic equilibrium at all. If $p \geq 1-\mathrm{e}^{-r U}$, then 0 is globally asymptotically stable and model (5) has globally asymptotically stable zero solution. If $p<1-\mathrm{e}^{-r U}$, then $x_{2}^{*}$ asymptotically attracts all positive solutions of (6) and model (5) has the globally asymptotically stable periodic solution $x(t)=\frac{K x_{2}^{*}}{x_{2}^{*}+\left(K-x_{2}^{*}\right) \mathrm{e}^{-r(t-n U)}}, t \in\left[n U^{+},(n+1) U\right], n=0,1,2, \ldots$

\subsection{Seasonally breeding model with lethal control}

This section deals with the seasonally breeding model with impulse lethal control. For convenience, suppose that lethal control is implemented at $n L T(n=0,1,2, \ldots)$ with removal rate $p$, that is, put the control in practice once every $L$ years and at the beginning of some breeding seasons. Thus, one can formulate model (7),

$$
\left\{\begin{array}{lll}
x^{\prime}=r_{1} x\left(1-\frac{x}{K}\right), & t \in\left[n T, n T+T_{1}\right], t \neq k L T, & \\
x^{\prime}=-r_{2} x, & t \in\left[n T+T_{1},(n+1) T\right], t \neq k L T, & n=0,1,2, \ldots, \\
x\left(k L T^{+}\right)=(1-p) x(k L T), & t=k L T, &
\end{array}\right.
$$

Solving model (7) and denoting $x\left(n L T^{+}\right)$by $X_{n}$ gives

$$
X_{n+1}=G_{3}\left(X_{n}\right)=\frac{(1-p) K X_{n} e^{-L r_{2} T_{2}}\left(e^{-r_{2} T_{2}}-e^{-r_{1} T_{1}}\right)}{X_{n}\left(1-e^{-r_{1} T_{1}}\right)\left(e^{-L r_{2} T_{2}}-e^{-L r_{1} T_{1}}\right)+K e^{-L r_{1} T_{1}}\left(e^{-r_{2} T_{2}}-e^{-r_{1} T_{1}}\right)} .
$$


Thus Theorem 3 is obtained from Lemma 1 and (8).

Theorem 30 is always an equilibrium of (8), when $p<1-e^{L\left(r_{2} T_{2}-r_{1} T_{1}\right)}$, then (8) has another unique positive equilibrium $x_{3}^{*}=\frac{K\left(e^{-r_{2} T_{2}}-e^{-r_{1} T_{1}}\right)\left[(1-p) e^{-L r_{2} T_{2}}-e^{-L r_{1} T_{1}}\right]}{\left(1-e^{-r_{1} T_{1}}\right)\left(e^{-L r_{2} T_{2}}-e^{-L r_{1} T_{1}}\right)}$. There is no other $h$ $(>1)$ periodic equilibrium at all. If $p \geq 1-e^{L\left(r_{2} T_{2}-r_{1} T_{1}\right)}$, then 0 is globally asymptotically stable and model (7) has globally asymptotically stable zero solution. If $p<1-e^{L\left(r_{2} T_{2}-r_{1} T_{1}\right)}$, then $x_{3}^{*}$ asymptotically attracts all positive solutions of (8) and model (7) has a globally asymptotically stable LT periodic solution.

\section{Impulse contraception control}

\subsection{Logistic model with contraception control}

Under contraception control, the rodent population is divided into a fertile subpopulation and a sterile subpopulation. Let $f(t)$ and $s(t)$ denote the sizes of the fertile subpopulation and the sterile subpopulation at time $t$, respectively. Assume that control is implemented once at regular time intervals $U$ and the contraception rate is $q$. The sterile individuals cannot produce offspring, but occupy resources normally. Denote the death rate of sterile subpopulation by $d$. Then one can formulate the logistic model with contraception control (9),

$$
\begin{cases}f^{\prime}=r f\left(1-\frac{f+s}{K}\right), & t \neq n U, \\ s^{\prime}=-d s, & t \neq n U, \\ f\left(n U^{+}\right)=(1-q) f(n U), & t=n U, \\ s\left(n U^{+}\right)=s(n U)+q f(n U), & t=n U .\end{cases}
$$

Solving model (9) on the interval $\left[n U^{+},(n+1) U^{+}\right]$, one can obtain the mapping (10) from $f\left(n U^{+}\right), s\left(n U^{+}\right)$to $f\left((n+1) U^{+}\right), s\left((n+1) U^{+}\right)$,

$$
\left\{\begin{array}{l}
f\left((n+1) U^{+}\right)=\frac{(1-q) K f\left(n U^{+}\right) \pi\left(U, s\left(n U^{+}\right)\right)}{K \pi\left(0, s\left(n U^{+}\right)\right)+r f\left(n U^{+}\right) \int_{0}^{U} \pi\left(v, s\left(n U^{+}\right)\right) d v}, \\
s\left((n+1) U^{+}\right)=s\left(n U^{+}\right) e^{-d U}+\frac{q K f\left(n U^{+}\right) \pi\left(U, s\left(n U^{+}\right)\right)}{K \pi\left(0, s\left(n U^{+}\right)\right)+r f\left(n U^{+}\right) \int_{0}^{U} \pi\left(v, s\left(n U^{+}\right)\right) d v},
\end{array}\right.
$$

where $\pi(t, u)=\exp \left(r t+\frac{r u}{d K} \mathrm{e}^{-d t}\right)$.

Theorem $4(0,0)$ is always an equilibrium of $(10)$, if $q<1-\mathrm{e}^{-r U}$, then (10) has another unique positive equilibrium $\left(f^{*}, s^{*}\right)$ such that $f^{*}=\frac{(1-q)\left(1-\mathrm{e}^{-d U}\right) s^{*}}{q}, s^{*}<s_{0}$ and $s_{0}=\frac{q(r+d) K}{r\left[1-(1-q) \mathrm{e}^{-d u}\right]}$.

Proof It is easy to see that $(0,0)$ is an equilibrium of $(10)$ and another equilibrium $(f, s)$ of (10) satisfies (11),

$$
\left\{\begin{array}{l}
1=\frac{(1-q) K \pi(U, s)}{K \pi(0, s)+r f \int_{0}^{U} \pi(v, s) d v} \\
s=s e^{-d U}+\frac{q K f \pi(U, s)}{K \pi(0, s)+r f \int_{0}^{U} \pi(v, s) d v}
\end{array}\right.
$$

Taking account the first equation in (11), one can rewrite the second equation in (11) as $f=\frac{(1-q)\left(1-\mathrm{e}^{-d U}\right) s}{q}$. Substituting this new relation into the first equation in (11) gives

$$
\varphi(s) \triangleq(1-q) K \pi(U, s)-K \pi(0, s)-\frac{r(1-q)\left(1-\mathrm{e}^{-d U}\right) s}{q} \int_{0}^{U} \pi(v, s) d v=0 .
$$


Because $\varphi(0)=K \mathrm{e}^{r U}\left(1-q-e^{-r U}\right)$, so if $q<1-\mathrm{e}^{-r U}$, then $\varphi(0)>0$, if $q \geq 1-\mathrm{e}^{-r U}$, then $\varphi(0) \leq 0$.

When $q \geq 1-\mathrm{e}^{-r U},(1-q) \mathrm{e}^{r U} \leq 1$ holds. Note that $\exp \left(\frac{r s}{d K} \mathrm{e}^{-d U}\right)<\exp \left(\frac{r s}{d K}\right)$, hence $(1-$ q) $K e^{r U} \exp \left(\frac{r s}{d K} \mathrm{e}^{-d U}\right)<K \exp \left(\frac{r s}{d K}\right)$, that is, $(1-q) K \pi(U, s)-K \pi(0, s)<0$. Thus $\varphi(s)<0$ for all $s>0$, and then $\varphi(s)=0$ has no positive solution.

Next, consider the existence of a positive solution of $\varphi(s)=0$ under the condition $q<$ $1-\mathrm{e}^{-r U}$. The fact that $\lim _{s \rightarrow+\infty}\left[r U+\frac{r s}{d K}\left(\mathrm{e}^{-d U}-1\right)\right]=-\infty$ implies

$$
\lim _{s \rightarrow+\infty}\left\{(1-q) \exp \left[r U+\frac{r s}{d K}\left(\mathrm{e}^{-d U}-1\right)\right]-1\right\}=-1
$$

This result, together with the fact that $\lim _{s \rightarrow+\infty} \exp \left(\frac{r s}{d K}\right)=+\infty$ gives

$$
\lim _{s \rightarrow+\infty} \exp \left(\frac{r s}{d K}\right)\left\{(1-q) \exp \left[r U+\frac{r s}{d K}\left(\mathrm{e}^{-d U}-1\right)\right]-1\right\}=-\infty
$$

that is, when $s \rightarrow+\infty$, the sum of the first two terms of $\varphi(s)$ approaches $-\infty$. Obviously, when $s \rightarrow+\infty$, the third term of $\varphi(s)$ approaches $-\infty$. So, $\lim _{s \rightarrow+\infty} \varphi(s)=-\infty$ and then when $q<1-\mathrm{e}^{-r U}, \varphi(s)=0$ has positive solution.

When $q<1-\mathrm{e}^{-r U}$, let $\mathrm{s}^{*}$ be a positive solution of $\varphi(s)=0$, then $\varphi\left(s^{*}\right)=0$ holds, that is,

$$
(1-q) K \pi\left(U, s^{*}\right)-K \pi\left(0, s^{*}\right)=\frac{r(1-q)\left(1-\mathrm{e}^{-d U}\right) s^{*}}{q} \int_{0}^{U} \pi\left(v, s^{*}\right) d v>0 .
$$

Because

$$
\begin{aligned}
r \int_{0}^{U} \pi\left(v, s^{*}\right) d v & =\int_{0}^{U} \exp \left(\frac{r s^{*}}{d K} \mathrm{e}^{-d v}\right) d \mathrm{e}^{r v} \\
& =\pi\left(T, s^{*}\right)-\pi\left(0, s^{*}\right)+\frac{r s^{*}}{K} \int_{0}^{U} \mathrm{e}^{-d v} \pi\left(v, s^{*}\right) d v
\end{aligned}
$$

we have

$$
\frac{r s^{*}}{K} \int_{0}^{U} \mathrm{e}^{-d v} \pi\left(v, s^{*}\right) d v=r \int_{0}^{U} \pi\left(v, s^{*}\right) d v-\pi\left(U, s^{*}\right)+\pi\left(0, s^{*}\right) .
$$

Thus the derivative of $\varphi$ with respect to $s$ at $s^{*}$ is

$$
\varphi^{\prime}\left(s^{*}\right)=\frac{r\left[1-(1-q) \mathrm{e}^{-d U}\right]}{q d s^{*}}\left[(1-q) \pi\left(U, s^{*}\right)-\pi\left(0, s^{*}\right)\right]\left(s^{*}-s_{0}\right) .
$$

Therefore, when $s^{*}>s_{0}, \varphi^{\prime}\left(s^{*}\right)>0$ holds; when $s^{*}<s_{0}, \varphi^{\prime}\left(s^{*}\right)<0$ holds; when $s^{*}=s_{0}$, $\varphi^{\prime}\left(s^{*}\right)=0$ holds.

If there exists $s^{*}$ that is larger than $s_{0}$, and $s_{1}$ is the maximum one of all $s^{*}$, then $\varphi^{\prime}\left(s_{1}\right)>0$. Thus there is a point $\bar{s}_{1}$ in a certain right neighborhood of $s_{1}$ such that $\bar{s}_{1}>s_{1}$ and $\varphi\left(\bar{s}_{1}\right)>0$. From the result of $\lim _{s \rightarrow+\infty} \varphi(s)=-\infty$, one knows that $\varphi(s)=0$ has a solution which is 
larger than $\bar{s}_{1}$. This is contrary to $s_{1}$ being maximum. Accordingly, $\varphi(s)$ does not have a zero point that is larger than $s_{0}$.

If there are at least two zero points of $\varphi(s)$ that are less than $s_{0}$ then one can select two points among all these zero points and denote them by $s_{2}$, $s_{3}$. So $\varphi^{\prime}\left(s_{2}\right)<0, \varphi^{\prime}\left(s_{3}\right)<0$. Without loss of generality, suppose $s_{2}<s_{3}$. Thus there is a point $\bar{s}_{2}$ in a certain right neighborhood of $s_{2}$ and a point $\bar{s}_{3}$ in a certain left neighborhood of $s_{3}$ such that $\bar{s}_{2}<\bar{s}_{3}, \varphi\left(\bar{s}_{2}\right)<0$ and $\varphi\left(\bar{s}_{3}\right)>0$. So there exists a zero point of $\varphi(s)$ between $\bar{s}_{2}$ and $\bar{s}_{3}$, thereby between $s_{2}$ and $s_{3}$. This result shows that there is at least one zero point of $\varphi(s)$ between any two zero points of it. So, the zero point of $\varphi(s)$ between $s_{2}$ and $s_{3}$ is dense. Then $\varphi^{\prime}\left(s_{2}\right)$ (or $\varphi^{\prime}\left(s_{3}\right)$ ) is 0 or does not exist, this is contrary to the fact that $\varphi^{\prime}\left(s_{2}\right)<0$ (or $\left.\varphi^{\prime}\left(s_{3}\right)<0\right)$. Therefore $\varphi(s)$ has no more than one zero point that is less than $s_{0}$.

If $s^{*}=s_{0}$, then $\varphi\left(s_{0}\right)=\varphi^{\prime}\left(s_{0}\right)=0$. Due to

$$
\begin{aligned}
(r-d) \int_{0}^{U} \pi\left(v, s^{*}\right) e^{-d v} d v & =\int_{0}^{U} \exp \left(\frac{r s^{*}}{d K} \mathrm{e}^{-d v}\right) d \mathrm{e}^{(r-d) v} \\
& =\pi\left(U, s^{*}\right) e^{-d U}-\pi\left(0, s^{*}\right)+\frac{r s^{*}}{K} \int_{0}^{U} \mathrm{e}^{-2 d v} \pi\left(v, s^{*}\right) d v,
\end{aligned}
$$

we have $\frac{r r^{*}}{K} \int_{0}^{U} \mathrm{e}^{-2 d v} \pi\left(v, s^{*}\right) d v=(r-d) \int_{0}^{U} \pi\left(v, s^{*}\right) e^{-d v} d v-\pi\left(U, s^{*}\right) e^{-d U}+\pi\left(0, s^{*}\right)$, and then

$$
\varphi^{\prime \prime}\left(s_{0}\right)=\frac{r^{2}\left[1-(1-q) \mathrm{e}^{-d U}\right]^{2}}{d K q^{2}(r+d)}\left[(1-q) \pi\left(U, s_{0}\right)-\pi\left(0, s_{0}\right)\right]>0 .
$$

Hence, $\varphi(s)$ takes a local minimum value at point $s_{0}$. Then there is a point $\bar{s}_{0}$ in a certain right neighborhood of $s_{0}$ such that $\bar{s}_{0}>s_{0}, \varphi\left(\bar{s}_{0}\right)>0$. This result, together with $\lim _{s \rightarrow+\infty} \varphi(s)=-\infty$, implies that $\varphi(s)$ has zero point that is larger than $\bar{s}_{0}$, thereby larger than $s_{0}$. This is contrary to the fact that $\varphi(s)$ does not have zero point that is larger than $s_{0}$. Consequently, $s_{0}$ is not zero point of $\varphi(s)$.

Summarily, $\varphi(s)$ has an unique positive zero point $s^{*}$ such that $s^{*}<s_{0}$. The proof is complete.

Theorem 5 If $q \geq 1-\mathrm{e}^{-r L}$, the equilibrium $(0,0)$ of $(10)$ is globally asymptotically stable and model (9) has a globally asymptotically stable zero solution.

Proof It is easy to see that $f\left(n U^{+}\right)>0, s\left(n U^{+}\right)>0$ for $n \in N=\{0,1,2,3, \ldots\}$ when $f\left(0^{+}\right)>0$, $s\left(0^{+}\right)>0$. The condition $(1-q) \mathrm{e}^{r U} \leq 1$ and the facts $\exp \left(\frac{r s\left(n U^{+}\right)}{d K} \mathrm{e}^{-d U}\right)<\exp \left(\frac{r s\left(n U^{+}\right)}{d K}\right)$, $r f\left(n U^{+}\right) \int_{0}^{U} \pi\left(v, S\left(n U^{+}\right)\right) d v>0$ imply $0<(1-q) K \pi\left(U, S\left(n U^{+}\right)\right)<K \pi\left(0, S\left(n U^{+}\right)\right)+$ $r f\left(n U^{+}\right) \int_{0}^{U} \pi\left(v, S\left(n U^{+}\right)\right) d v$. This indicates that $f\left((n+1) U^{+}\right)<f\left(n U^{+}\right)$, that is, the sequence $\left\{f\left(n U^{+}\right)\right\}$is descending. Since 0 is the lower bound of $\left\{f\left(n U^{+}\right)\right\}$, sequence $\left\{f\left(n U^{+}\right)\right\}$converges and its limit is larger than or equals 0 .

If there exists $k$ such that $s\left((k+1) U^{+}\right)<s\left(k U^{+}\right)$, then $s\left((n+1) U^{+}\right)=s\left(n U^{+}\right) e^{-d U}+\frac{q f\left((n+1) U^{+}\right)}{1-q}$ and $\left\{f\left(n U^{+}\right)\right\}$being descending imply $s\left((n+1) U^{+}\right)<s\left(n U^{+}\right)$for all $n \geq k$, that is, the sequence $\left\{s\left(n U^{+}\right)\right\}$is descending for $n \geq k$. Since 0 is the lower bound of $\left\{s\left(n U^{+}\right)\right\}$, the sequence $\left\{s\left(n U^{+}\right)\right\}$converges.

If there is no $k$ such that $s\left((k+1) U^{+}\right)<s\left(k U^{+}\right)$, namely, the sequence $\left\{s\left(n U^{+}\right)\right\}$is ascending, then from the relation $s\left(n U^{+}\right) \leq s\left((n+1) U^{+}\right)=s\left(n U^{+}\right) e^{-d U}+\frac{q f\left((n+1) U^{+}\right)}{1-q}$, one can 
conclude $s\left(n U^{+}\right) \leq \frac{q f\left((n+1) U^{+}\right)}{(1-q)\left(1-\mathrm{e}^{-d U}\right)}$, that is, $\left\{s\left(n U^{+}\right)\right\}$is bounded. Thus, the sequence $\left\{s\left(n U^{+}\right)\right\}$ converges.

The sequence $\left\{s\left(n U^{+}\right)\right\}$always converges.

Let $\lim _{n \rightarrow+\infty} f\left(n U^{+}\right)=A$ and $\lim _{n \rightarrow+\infty} s\left(n U^{+}\right)=B$. Taking limits at both sides of the two equations in (10) gives

$$
\left\{\begin{array}{l}
A=\frac{(1-q) K A \pi(U, B)}{K \pi(0, B)+r A \int_{0}^{U} \pi(v, B) d v}, \\
B=B e^{-d U}+\frac{q K A \pi(U, B)}{K \pi(0, B)+r A \int_{0}^{U} \pi(v, B) d v} .
\end{array}\right.
$$

Form the first formula in (13), one obtains $A=0$ or $A=\frac{(1-q) K A \pi(U, B)-K \pi(0, B)}{r \int_{0}^{U} \pi(v, B) d v}$. Because the latter result is less than 0 and $A \geq 0$, we have $A=0$. Afterwards, it is easy to see that $B=0$ from the second formula in (13).

Therefore, the equilibrium $(0,0)$ of $(10)$ is globally asymptotically stable.

\section{Theorem 6 Let}

$$
\begin{aligned}
\Lambda= & \frac{1}{(1-q) \pi\left(U, s^{*}\right)}\left\{\left(1+\mathrm{e}^{-d U}\right)\left[(1-q) \pi\left(U, s^{*}\right)+\pi\left(0, s^{*}\right)\right]\right. \\
& +\frac{r}{d K(1-q)}\left\{\left[1-(1-q) e^{-d U}\right] f^{*}-K(1-q)\left(1-e^{-d U}\right)\right\} \\
& \left.\times\left[(1-q) \pi\left(U, s^{*}\right)-\pi\left(0, s^{*}\right)\right]\right\} .
\end{aligned}
$$

If $q<1-\mathrm{e}^{-r U}$ and $\Lambda>0$, the positive equilibrium $\left(f^{*}, s^{*}\right)$ of $(10)$ is locally asymptotically stable.

Proof The linearized matrix of $(10)$ at $\left(f^{*}, s^{*}\right)$ is $J=\left[\begin{array}{ll}J_{11} & J_{12} \\ J_{21} J_{22}\end{array}\right]$, here $J_{11}=\frac{\pi\left(0, s^{*}\right)}{(1-q) \pi\left(T, s^{*}\right)}, J_{12}=$ $\frac{r}{d K q(1-q)}\left\{\left[1-(1-q) \mathrm{e}^{-d U}\right] f^{*}-(1-q)\left(1-\mathrm{e}^{-d U}\right) K\right\}\left[(1-q) \pi\left(U, s^{*}\right)-\pi\left(0, s^{*}\right)\right], J_{21}=\frac{q J_{11}}{(1-q)}, J_{22}=$ $\mathrm{e}^{-d U}+\frac{q J_{12}}{(1-q)}$.

The characteristic equation of $J$ is $\lambda^{2}+a_{1} \lambda+a_{2}=0$, here $a_{1}=-J_{11}-J_{22}, a_{2}=|J|=$ $\mathrm{e}^{-d U} \frac{\pi\left(0, s^{*}\right)}{(1-q) \pi\left(U, s^{*}\right)}$. Equation (12) tells us $(1-q) \pi\left(T, s^{*}\right)>\pi\left(0, s^{*}\right)>0$, so $0<a_{2}<1$. Since

$$
1+a_{1}+a_{2}=\frac{1-\mathrm{e}^{-d U}}{d(1-q) \pi\left(U, s^{*}\right)}\left[(1-q) \pi\left(U, s^{*}\right)-\pi\left(0, s^{*}\right)\right]\left[r+d-\frac{r\left[1-(1-q) e^{-d U}\right] s^{*}}{q K}\right]
$$

and $s^{*}<s_{0}$, we have $1+a_{1}+a_{2}>0$. Calculation shows $1-a_{1}+a_{2}=\Lambda>0$. Therefore, on the basis of the Jury criterion, the positive equilibrium $\left(f^{*}, s^{*}\right)$ of (10) is locally asymptotically stable.

\subsection{Seasonally breeding model with contraception control}

Next, consider impulse contraception control based on the seasonally breeding model (1). For convenience, suppose that contraception control is implemented at $n L T(n=0,1,2, \ldots)$ with contraception rate $q$, that is, put the control in practice once every $L$ years and at the 
beginning of some breeding seasons. Thus, one can formulate model (14),

$$
\begin{cases}f^{\prime}=r_{1} f\left(1-\frac{f+s}{K}\right), & t \in\left[n T, n T+T_{1}\right], t \neq k L T, \\ s^{\prime}=-d_{1} s, & t \in\left[n T, n T+T_{1}\right], t \neq k L T, \\ f^{\prime}=-r_{2} f, & t \in\left[n T+T_{1},(n+1) T\right], t \neq k L T, \quad n=0,1,2, \ldots, \\ s^{\prime}=-r_{2} s, & t \in\left[n T+T_{1},(n+1) T\right], t \neq k L T, \quad k=0,1,2, \ldots \\ f\left(k L T^{+}\right)=(1-q) f(k L T), & t=k L T, \\ s\left(k L T^{+}\right)=s(k L T)+q f(k L T), & t=k L T,\end{cases}
$$

Solving model (14) and denoting $f\left(n L T^{+}\right), s\left(n L T^{+}\right)$as $F_{n}, S_{n}$, respectively, gives

$$
\left\{\begin{array}{l}
F_{n+1}=\frac{(1-q) K F_{n} g_{1}\left(S_{n}\right)}{K+r_{1} g_{n} g_{2}\left(S_{n}\right)}, \\
S_{n+1}=S_{n} \mathrm{e}^{-L\left(d_{1} T_{1}+r_{2} T_{2}\right)}+\frac{q K F_{n} g_{1}\left(S_{n}\right)}{K+r_{1} F_{n} g_{2}\left(S_{n}\right)},
\end{array}\right.
$$

where

$$
\begin{aligned}
g_{1}\left(S_{n}\right)= & \exp \left\{L\left(r_{1} T_{1}-r_{2} T_{2}\right)+\frac{r_{1} S_{n}\left[1-e^{-L\left(d_{1} T_{1}+r_{2} T_{2}\right)}\right]}{d_{1} K\left(1-e^{-d_{1} T_{1}-r_{2} T_{2}}\right)}\left(\mathrm{e}^{-d_{1} T_{1}}-1\right)\right\}, \\
g_{2}\left(S_{n}\right)= & \int_{0}^{T_{1}} \exp \left[r_{1} v+\frac{r_{1} S_{n}}{d_{1} K}\left(\mathrm{e}^{-d_{1} v}-1\right)\right] d v+\exp \left[r_{1} T_{1}-r_{2} T_{2}+\frac{r_{1} S_{n}}{d_{1} K}\left(\mathrm{e}^{-d_{1} T_{1}}-1\right)\right] \\
& \times \int_{0}^{T_{1}} \exp \left[r_{1} v+\frac{r_{1} S_{n}}{d_{1} K}\left(\mathrm{e}^{-d_{1} v}-1\right) \mathrm{e}^{-d_{1} T_{1}-r_{2} T_{2}}\right] d v \\
& +\exp \left[2 r_{1} T_{1}-2 r_{2} T_{2}+\frac{r_{1} S_{n}}{d_{1} K}\left(\mathrm{e}^{-d_{1} T_{1}}-1\right)\left(1+\mathrm{e}^{-d_{1} T_{1}-r_{2} T_{2}}\right)\right] \\
& \times \int_{0}^{T_{1}} \exp \left[r_{1} v+\frac{r_{1} S_{n}}{d_{1} K}\left(\mathrm{e}^{-d_{1} v}-1\right) \mathrm{e}^{-2\left(d_{1} T_{1}+r_{2} T_{2}\right)}\right] d v \\
& +\cdots \\
& +\exp \left[(L-1)\left(r_{1} T_{1}-2 r_{2} T_{2}\right)\right. \\
& \left.+\frac{r_{1} S_{n}}{d_{1} K}\left(\mathrm{e}^{-d_{1} T_{1}}-1\right)\left(1+\mathrm{e}^{-d_{1} T_{1}-r_{2} T_{2}}+\cdots+\mathrm{e}^{-(L-2)\left(d_{1} T_{1}+r_{2} T_{2}\right)}\right)\right] \\
& \times \int_{0}^{T_{1}} \exp \left[r_{1} v+\frac{r_{1} S_{n}}{d_{1} K}\left(\mathrm{e}^{-d_{1} v}-1\right) \mathrm{e}^{-(L-1)\left(d_{1} T_{1}+r_{2} T_{2}\right)}\right] d v .
\end{aligned}
$$

Theorem $7(0,0)$ is always an equilibrium of (15), if $q<1-\mathrm{e}^{-L\left(r_{1} T_{1}-r_{2} T_{2}\right)}$, then (15) has another positive equilibrium.

Proof Obviously, $(0,0)$ is equilibrium of $(15)$ and other equilibrium $(F, S)$ of $(15)$ satisfies (16)

$$
\left\{\begin{array}{l}
1=\frac{(1-q) K g_{1}(S)}{K+r_{1} F g_{2}(S)}, \\
S=S \mathrm{e}^{-L\left(d_{1} T_{1}+r_{2} T_{2}\right)}+\frac{q F}{1-q} .
\end{array}\right.
$$


From the first equation in (16), one sees that $F=\frac{(1-q) K g_{1}(S)-K}{r_{1} g_{2}(S)}$. Substituting this new relation into the second equation in (16) gives

$$
0=\phi(S) \triangleq(1-q) K g_{1}(S)-K-\frac{(1-q) r_{1} K S g_{1}(S)}{q}\left[1-\mathrm{e}^{-L\left(d_{1} T_{1}+r_{2} T_{2}\right)}\right] .
$$

Note that $\phi(0)=K\left[(1-q) \mathrm{e}^{L\left(r_{1} T_{1}-r_{2} T_{2}\right)}-1\right]$, so if $q<1-\mathrm{e}^{-L\left(r_{1} T_{1}-r_{2} T_{2}\right)}$, then $\phi(0)>0$ and if $q \geq 1-\mathrm{e}^{-L\left(r_{1} T_{1}-r_{2} T_{2}\right)}$, then $\phi(0) \leq 0$.

When $q \geq 1-\mathrm{e}^{-L\left(r_{1} T_{1}-r_{2} T_{2}\right)},(1-q) \mathrm{e}^{L\left(r_{1} T_{1}-r_{2} T_{2}\right)} \leq 1$ holds. Hence the first term of $\phi(S)$ is less than $K$ and then $\phi(S)<0$ for arbitrary $S \geq 0$. This means that $\phi(S)=0$ does not have a positive solution.

When $q<1-\mathrm{e}^{-L\left(r_{1} T_{1}-r_{2} T_{2}\right)}$, the first term of $\phi(S)$ tends to 0 as $S$ approaching to $+\infty$, the second term is a negative constant, the third term is less than 0 . So when $S$ is large enough, $\phi(S)<0$ holds. It implies that $\phi(S)=0$ has a positive solution.

Theorem 8 If $q \geq 1-\mathrm{e}^{-L\left(r_{1} T_{1}-r_{2} T_{2}\right)}$, the equilibrium $(0,0)$ of $(15)$ is globally asymptotically stable and model (14) has a globally asymptotically stable zero solution.

Proof Obviously, when $F_{0}>0, S_{0}>0, F_{n}>0, S_{n}>0$ hold for $n \in N=\{0,1,2,3, \ldots\}$. When the stated conditions hold, $0<(1-q) \mathrm{e}^{L\left(r_{1} T_{1}-r_{2} T_{2}\right)} \leq 1, \frac{r_{1} S_{n}\left[1-e^{-L\left(d_{1} T_{1}+r_{2} T_{2}\right)}\right.}{d_{1} K\left(1-e^{\left.-d_{1} T_{1}-r_{2} T_{2}\right)}\right.}\left(\mathrm{e}^{-d_{1} T_{1}}-1\right)<0$, all these result in $0<(1-q) K g_{1}\left(S_{n}\right)<K$. In addition, $r_{1} F_{n} g_{2}\left(S_{n}\right)>0$, so $0<(1-q) K F_{n} g_{1}\left(S_{n}\right)<$ $K+r_{1} F_{n} g_{2}\left(S_{n}\right)$. Thus $F_{n+1}<F_{n}$, that is, $\left\{F_{n}\right\}$ is descending. Since 0 is the lower bound of $\left\{F_{n}\right\}$, the sequence $\left\{F_{n}\right\}$ converges and its limit is larger than or equals 0 .

If there exists $k$ such that $S_{k+1}<S_{k}$, then the facts that $S_{n+1}=S_{n} \mathrm{e}^{-L\left(d_{1} T_{1}+r_{2} T_{2}\right)}+\frac{q F_{n+1}}{1-q}$ and $\left\{F_{n}\right\}$ is descending imply $S_{n+1}<S_{n}$ for all $n \geq k$, that is, the sequence $\left\{S_{n}\right\}$ is descending for $n \geq k$. Since 0 is the lower bound of $\left\{S_{n}\right\}$, the sequence $\left\{S_{n}\right\}$ converges.

If there is no $k$ such that $S_{k+1}<S_{k}$, namely, the sequence $\left\{S_{n}\right\}$ is ascending, then, from the relation $S_{n} \leq S_{n+1}=S_{n} e^{-L\left(d_{1} T_{1}+r_{2} T_{2}\right)}+\frac{q F_{n+1}}{1-q}$, one can conclude $S_{n} \leq \frac{q F_{n+1}}{(1-q)\left[1-e^{-L\left(d_{1} T_{1}+r_{2} T_{2}\right)}\right]}$, that is, $\left\{S_{n}\right\}$ is bounded. Thus, the sequence $\left\{S_{n}\right\}$ converges.

Thus, the sequence $\left\{S_{n}\right\}$ always converges.

Assume that $\lim _{n \rightarrow+\infty} F_{n}=A$ and $\lim _{n \rightarrow+\infty} S_{n}=B$. Taking limits at both sides of the two equations in (15) gives

$$
\left\{\begin{array}{l}
A=\frac{(1-q) K A g_{1}(B)}{K+r_{1} A g_{2}(B)}, \\
B=B \mathrm{e}^{-L\left(d_{1} T_{1}+r_{2} T_{2}\right)}+\frac{q K A g_{1}(B)}{K+r_{1} A g_{2}(B)} .
\end{array}\right.
$$

From the first formula in (18), one obtains $A=0$ or $A=\frac{(1-q) K g_{1}(B)-K}{r_{1} g_{2}(B)}$. The latter result is less than 0 and $A \geq 0$, so $A=0$. Afterwards, it is easy to see that $B=0$ from the second formula in (18).

Therefore, the equilibrium $(0,0)$ of $(15)$ is globally asymptotically stable.

\section{Theorem 9 Denote}

$$
\begin{aligned}
\Lambda_{1}= & 1-\frac{1}{(1-q) g_{1}\left(S^{*}\right)}-\mathrm{e}^{-L\left(d_{1} T_{1}+r_{2} T_{2}\right)} \\
& -\frac{(1-q) q K F^{*} g_{1}^{\prime}\left(S^{*}\right)-q r_{1}\left(F^{*}\right)^{2} g_{2}^{\prime}\left(S^{*}\right)}{(1-q)^{2} K g_{1}\left(S^{*}\right)}+\frac{\mathrm{e}^{-L\left(d_{1} T_{1}+r_{2} T_{2}\right)}}{(1-q) g_{1}\left(S^{*}\right)},
\end{aligned}
$$




$$
\begin{aligned}
\Lambda_{2}= & +\frac{1}{(1-q) g_{1}\left(S^{*}\right)}+\mathrm{e}^{-L\left(d_{1} T_{1}+r_{2} T_{2}\right)} \\
& +\frac{(1-q) q K F^{*} g_{1}^{\prime}\left(S^{*}\right)-q r_{1}\left(F^{*}\right)^{2} g_{2}^{\prime}\left(S^{*}\right)}{(1-q)^{2} K g_{1}\left(S^{*}\right)}+\frac{\mathrm{e}^{-L\left(d_{1} T_{1}+r_{2} T_{2}\right)}}{(1-q) g_{1}\left(S^{*}\right)} .
\end{aligned}
$$

When $\Lambda_{1}>0, \Lambda_{2}>0, q<1-\mathrm{e}^{-L\left(r_{1} T_{1}-r_{2} T_{2}\right)}$ hold, the positive equilibrium of (15) is locally asymptotically stable.

Proof The linearized matrix of the map (15) at positive equilibrium $\left(F^{*}, S^{*}\right)$ is $M=$ $\left[\begin{array}{ll}M_{11} & M_{12} \\ M_{21} & M_{22}\end{array}\right]$ with $M_{11}=\frac{1}{(1-q) g_{1}\left(S^{*}\right)}, M_{12}=\frac{(1-q) K F^{*} g_{1}^{\prime}\left(S^{*}\right)-r_{1}\left(F^{*}\right)^{2} g_{2}^{\prime}\left(S^{*}\right)}{(1-q) K g_{1}\left(S^{*}\right)}, M_{21}=\frac{q}{1-q} M_{11}, M_{22}=$ $\mathrm{e}^{-L\left(d_{1} T_{1}+r_{2} T_{2}\right)}+\frac{q}{1-q} M_{12}$.

The characteristic equation of $M$ is $\lambda^{2}+b_{1} \lambda+b_{2}=0$, here $b_{1}=-M_{11}-M_{22}, b_{2}=|M|=$ $\frac{\mathrm{e}^{-L\left(d_{1} T_{1}+r_{2} T_{2}\right)}}{(1-q) g_{1}\left(S^{*}\right)}$. Equation (17) tells us $(1-q) g_{1}\left(S^{*}\right)>1$, so $0<b_{2}<1$. Simple calculation shows $1-b_{1}+b_{2}=\Lambda_{1}>0,1+b_{1}+b_{2}=\Lambda_{2}>0$. Therefore, on the basis of the Jury criterion, the positive equilibrium of (15) is locally asymptotically stable.

\section{Numerical simulation}

The foregoing analysis shows that the condition of the rodent population dying out is approximately the same for lethal control and contraception control. For the logistic model with lethal control (5) and the logistic model with contraception control (10), when the control rate $(p$ or $q)$ is larger than $1-\mathrm{e}^{-r U}$, the rodent dies out, and when the control rate ( $p$ or $q$ ) is smaller than 1- $\mathrm{e}^{-r U}$, the rodent persists. For the seasonally breeding model with lethal control (7) and the seasonally breeding model with contraception control (14), when the control rate $(p$ or $q)$ is larger than 1- $\mathrm{e}^{L\left(r_{2} T_{2}-r_{1} T_{1}\right)}$, the rodent dies out, and when the control rate $(p$ or $q)$ is smaller than $1-\mathrm{e}^{L\left(r_{2} T_{2}-r_{1} T_{1}\right)}$, the rodent persists. However, these two controls are different, the process of the rodent population tending to a stable solution obviously is different (Figures 1 and 2).

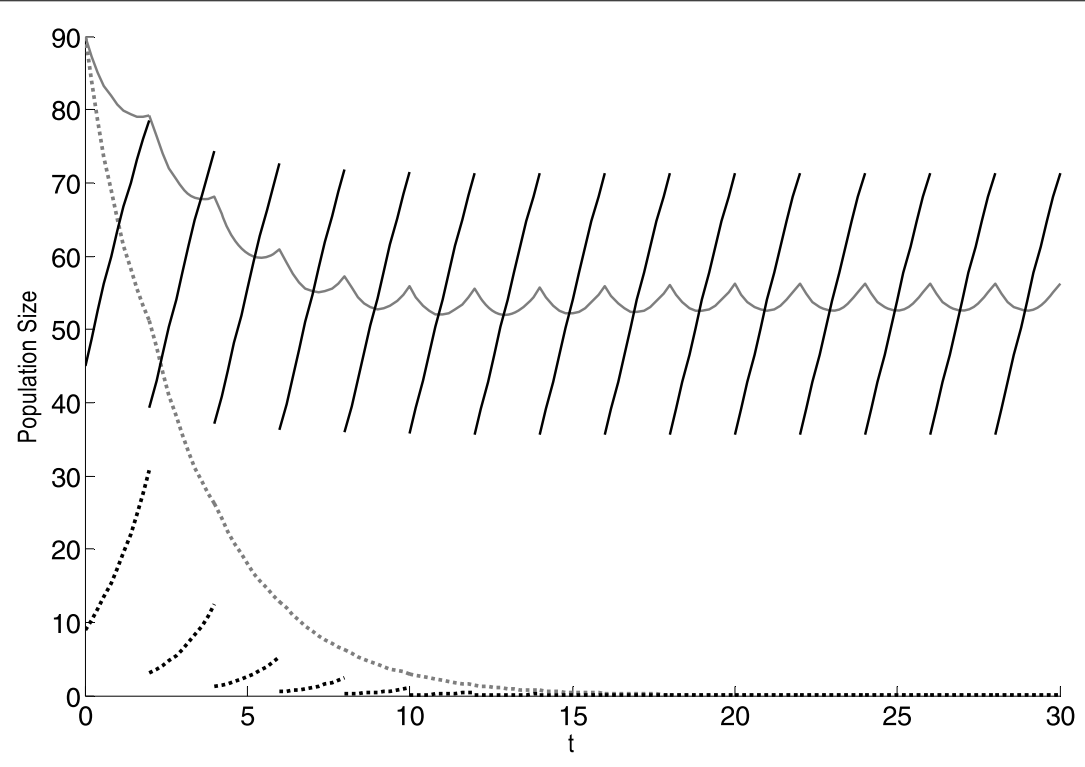

Figure 1 The dynamics of rodent population in model (5) and (10). 


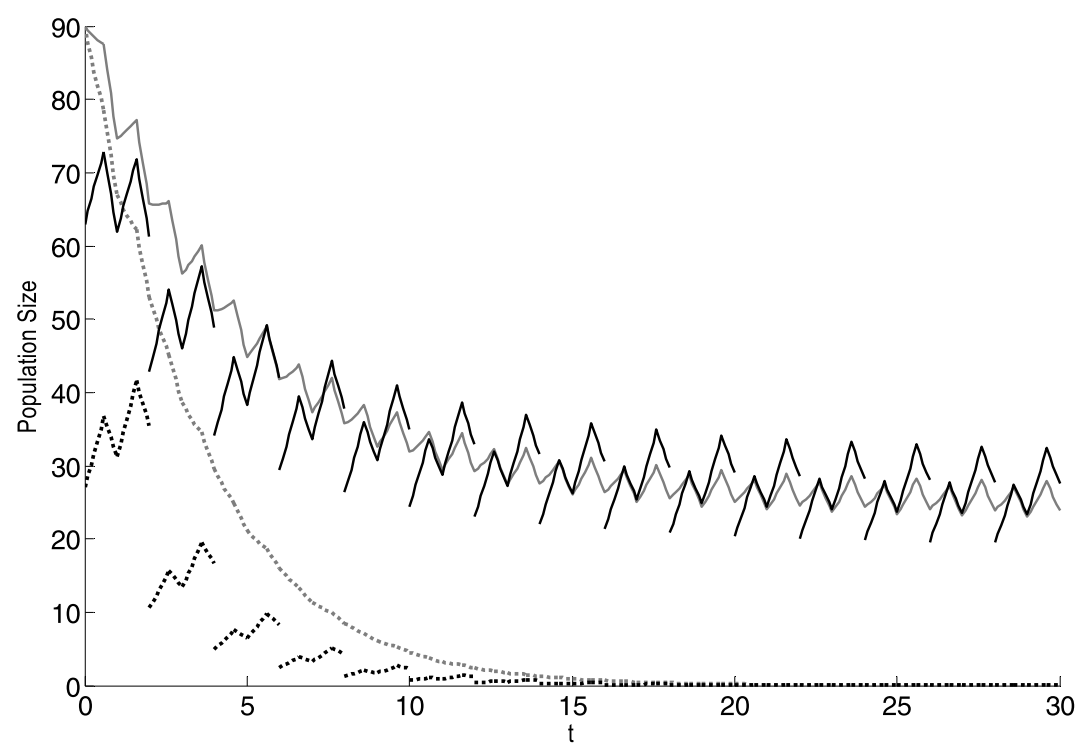

Figure 2 The dynamics of rodent population in model (7) and (14).

Figure 1 shows the dynamics of the rodent population in model (5) and (10), where $r=$ $0.75, K=100, d=0.4, U=2$. For the solid line, the control rate ( $p$ or $q$ ) is 0.5 , whereas, for the dotted line, it is 0.9 . Figure 2 shows the dynamics of rodent population in model (7) and (14), where $r_{1}=0.75, K=100, r_{2}=0.4, T_{1}=0.6, T_{2}=0.4$. For the solid line, the control rate ( $p$ or $q)$ is 0.3 , whereas, for the dotted line, it is 0.7 . In both figures, the black line and gray line refer to the dynamics of the rodent population with lethal control and contraception control, respectively, and the initial value is $x(0)=90$ or $f(0)=90, s(0)=0$.

Under lethal control, the rodent population approaches a stable periodic solution more quickly. So, if it was urgent to wipe off the rodent, the lethal control would be more effective. Under contraception control, the rodent population develops slowly. The variation of the rodent population is one kind of disturbance to the ecosystem, if this disturbance went beyond the tolerance of an ecosystem, the ecosystem would be disorganized, even it might collapse. Thus, for fragile ecosystems, contraception control is appropriate.

\section{Discussion}

In this paper, dynamics models are formulated based on the logistic model and the seasonally breeding model to investigate the effect of impulse lethal and contraception control on rodent population dynamics. The existence and stability of the periodic solution are analyzed. The condition of the rodent population dying out is the same for these two controls and each control has its own traits.

If the rodent control can wipe it off thoroughly then there would never exist damage in the future. However, this situation is awful. Among all biotic and abiotic components of an ecosystem, there are complicated relations. If the rodent dies out, the dynamics of the other components would vary correspondingly. When these variations are large enough, the ecosystem would be disorganized, even collapse and result in greater damage. Thus, the aim of rodent control is guiding rodent population to a suitable size, not exterminating it. For example, the plateau pika brings great damage to degraded alpine meadow, but it is a keystone species also $[16,17]$. The extinction of plateau pika may reduce the loss of pasture 
grasses that plateau pika feeds on, but, most importantly, this may induce extinction of some raptors that prey on plateau pika, and it may induce extinction of some birds that inhibit holes of plateau pika.

For an epidemic model, there is an important parameter, namely, the basic reproductive number $R_{0}$. Usually, the disease-free equilibrium is globally asymptotically stable if $R_{0}<1$ and the endemic equilibrium is globally asymptotically stable if $R_{0}>1$. For population dynamics model, frequently, when some conditions hold there is a stable positive equilibrium, otherwise there is a stable zero equilibrium. From these conditions, one can dig out a parameter that is similar to $R_{0}$. Denote this parameter by $R_{0}$. In this paper, $R_{0}$ is the maximum finite rate of increase during a specific time interval. Consider a population of which the size satisfies the Malthus model $x^{\prime}=r x$, and suppose its size is $x_{0}$ at time $t_{0}$. Then its solution is $x(t)=x_{0} \mathrm{e}^{r\left(t-t_{0}\right)}$, and after a time interval $T$, that is, at time $t_{0}+T$, the population size is $x\left(t_{0}+T\right)=x_{0} \mathrm{e}^{r T}$. So during this time interval of length $T$, the multiplication factor of the population size is $\mathrm{e}^{r T}$, and this is the finite rate of increase during this time interval.

In the seasonally breeding model (1), the condition of a positive equilibrium existing and being stable is $r_{2} T_{2}<r_{1} T_{1}$, namely, $\mathrm{e}^{r_{1} T_{1}} \mathrm{e}^{-r_{2} T_{2}}>1$. Model (1) is a periodic system with 1 -year period. For the breeding season, its length is $T_{1}$ and the maximum increasing rate is $r_{1}$, so the maximum finite rate of increase is $\mathrm{e}^{r_{1} T_{1}}$. For the non-breeding season, its length is $T_{2}$ and the maximum increasing rate is $-r_{2}$, so the maximum finite rate of increase is $\mathrm{e}^{-r_{2} T_{2}}$. Therefore, during one period, the maximum finite rate of increase is $\mathrm{e}^{r_{1} T_{1}} \mathrm{e}^{-r_{2} T_{2}}$, and this is the quantity $R_{0}$. In model (9), which is derived from the logistic model and in which the rodent are subject to impulse contraception control, the condition of positive equilibrium existing and being stable is $q<1-\mathrm{e}^{-r U}$, namely, $(1-q) \mathrm{e}^{r U}>1$. Consider one control period of length $U$ and assume that control is implemented at the beginning of this period, so the ratio of fertile individuals to all individuals is $1-q$. During one time interval of length $U$, the maximum finite rate of increase is $\mathrm{e}^{r U}$, and then during one control period, the maximum finite rate of increase is $(1-q) \mathrm{e}^{r U}$, and this is $R_{0}$. For other models, one can obtain the maximum finite rate of increase in a similar way and see that it is just $R_{0}$.

Competing interests

The authors declare that they have no competing interests.

Authors' contributions

$\mathrm{HL}$ designed and performed all the steps of the proofs in this research. FZ and QL participated in the design of the study and suggested many good ideas that made this paper possible. FZ performed the simulation. QL wrote the paper. All authors read and approved the final manuscript.

Acknowledgements

This work is partially supported by the National Natural Science Foundation of China under Grants No. 11371313, 61573016,61203228 , the Natural Science Foundation of Shanxi Province under Grant No. 2013011002-5.

\section{Publisher's Note}

Springer Nature remains neutral with regard to jurisdictional claims in published maps and institutional affiliations.

Received: 14 December 2016 Accepted: 20 March 2017 Published online: 29 March 2017

References

1. Liu, H, Jin, Z, Chen, Y, Zhang, F: Population dynamics of plateau pika under lethal control and contraception control. Adv. Differ. Equ. (2012). doi:10.1186/1687-1847-2012-29

2. Liu, H, Jin, Z, Zhang, F, Li, Q: Dynamic model of seasonal breeding rodent pest population controlled with short-acting sterilant. Chin. J. Appl. Ecol. 24, 1141-1145 (2013)

3. Bronson, FH: Mammalian reproduction: an ecological perspective. Biol. Reprod. 32, 1-26 (1985) 
4. Bronson, FH, Perrigo, G: Seasonal regulation of reproduction in muroid rodents. Am. Zool. 27, 929-940 (1987)

5. Wang, X, Dai, K: Studies on the population reproduction ecology of plateau pika. Zool. Res. 12, 155-161 (1991)

6. Sicard, B, Fuminier, F: Environmental cues and seasonal breeding patterns in Sahelian rodents. Mammalia 60, 667-675 (1996)

7. Spinks, AC, Van der Horst, G, Bennett, NC: Influence of breeding season and reproductive status on male reproductive characteristics in the common mole-rat, Cryptomys hottentotus hottentotus. J. Reprod. Fertil. 109, 79-86 (1997)

8. Janse van Rensburg, L, Bennett, NC, Van der Merwe, M, Schoeman, AS: Seasonal reproduction in the highveld mole-rat, Cryptomys hottentotus pretoriae (Rodentia: Bathyergidae). Can. J. Zool. 80, 810-820 (2002)

9. Herbst, M, Jarvis, JUM, Bennett, NC: A field assessment of reproductive seasonality in the threatened wild Namaqua dune mole-rat (Bathyergus janetta). J. Zool. 263, 259-268 (2004)

10. Makundi, RH, Massawe, AW, Mulungu, LS: Breeding seasonality and population dynamics of three rodent species in the Magamba Forest Reserve, Western Usambara Mountains, north-east Tanzania. Afr. J. Ecol. 45, 17-21 (2007)

11. Wang, D, Cong, L, Wang, Y, Liu, X: Population parameters and physiological characteristics of Brandt's vole (Lasiopodomys brandtii) in breeding and non-breeding seasons. Acta Ecol. Sin. 30, 3562-3568 (2010)

12. Liu, W, Wan, X, Zhong, W, Wang, J: Characteristics of seasonal reproduction in Mongolian gerbils (Meriones unguiculatus). Acta Theriol. Sin. 33, 35-46 (2013)

13. Sarli, J, Lutermann, H, Alagaili, AN, Mohammed, OB, Bennett, NC: Seasonal reproduction in the Arabian spiny mouse, Acomys dimidiatus (Rodentia: Muridae) from Saudi Arabia: the role of rainfall and temperature. J. Arid Environ. 124 352-359 (2016)

14. Rocha, CR, Ribeiro, R, Marinho-Filho, J: Influence of temporal variation and seasonality on population dynamics of three sympatric rodents. Mamm. Biol. 84, 20-29 (2017)

15. Liu, H, Zhou, L, Liu, W, Zhou, H: Dynamic model for population whose growing and non-growing season present in turns and the influence of environmental change. J. Biomath. 23, 435-442 (2008)

16. Smith, AT, Foggin, JM: The plateau pika (Ochotona curzoniae) is a keystone species for biodiversity on the Tibetan plateau. Anim. Conserv. 2, 235-240 (1999)

17. Lai, CH, Smith, AT: Keystone status of plateau pikas (Ochotona curzoniae): effect of control on biodiversity of native birds. Biodivers. Conserv. 12, 1901-1912 (2003)

\section{Submit your manuscript to a SpringerOpen ${ }^{\circ}$ journal and benefit from:}

- Convenient online submission

- Rigorous peer review

- Immediate publication on acceptance

- Open access: articles freely available online

- High visibility within the field

- Retaining the copyright to your article 